\title{
Monitoring Heat-Related Illness through Syndromic Surveillance in Los Angeles County
}

\section{Jimmy Duong, Michael Lim, Emily Kajita, Bessie Hwang}

Public Health, Los Angeles County, Los Angeles, California, United States

Objective

To analyze Los Angeles County's (LAC) extreme heat season in 2018 and evaluate the Council of State and Territorial Epidemiologists' (CSTE) syndrome query for heat-related-illness (HRI) in Los Angeles County (LAC)

\section{Introduction}

LAC experienced several days of record-breaking temperatures during the summer of 2018. Downtown Los Angeles temperatures soared to $108^{\circ} \mathrm{F}$ in July with an average daily maximum of $92^{\circ} \mathrm{F}$. Extreme heat events such as these can pose major risks to human health. Syndromic surveillance can be a useful tool in providing near real-time surveillance of HRI. In 2014, a working group was formed within the CSTE Climate Change Subcommittee to define and analyze HRI. The workgroup's goal was to provide guidance to public health professionals in adapting and implementing an HRI syndrome surveillance query. The Acute Communicable Disease Control Program's (ACDC) Syndromic Surveillance Unit utilized CSTE's HRI query to provide surveillance during the extreme heat season in 2018 in LAC. Additional modifications to the CSTE query were evaluated for potential improvements towards characterizing HRI trends.

\section{Methods}

From May 1 to September 30, 2018, Emergency Department (ED) data were queried for cases using the CSTEs definition for HRI. The queries consisted of key word searches within the chief complaint (CC) data field, and, if available, the diagnosis data fields. The query was derived from the CSTE HRI query published in 2016 [1]. In addition, ACDC explored the utility of expanding the CSTE syndrome definition to include additional chief complaints commonly associated with HRI such as dehydration and syncope. Both queries were applied on all participating syndromic EDs in LAC alongside daily high temperature data trends.

Local temperature data for downtown Los Angeles weather station KCQT were taken from the Weather Underground website. Spearman correlation coefficients were calculated for each query during the heat season. Similarly, both queries were also applied during colder months from October 1, 2017 to April 30, 2018 for comparison. Lastly, results for dehydration and syncope were independently assessed apart from other HRI query terms during both heat seasons and colder months.

\section{Results}

The CSTE HRI query and the query with the added terms yielded 1,258 and 63,332 ED visits, respectively, during the heat season. On July 6 , the maximum daily temperature peaked at $108^{\circ} \mathrm{F}$; the HRI and the query with the added terms yielded 136 and 618 ED visits, respectively. The HRI query and the HRI query with the added terms had a correlation coefficient of 0.714 ( $p<0.0001)$ and 0.427 ( $\mathrm{p}<0.0001$ ), respectively. During colder months, the CSTE HRI query and the query with the added terms yielded 377 and 86,008 , respectively, with correlation coefficients of $0.342(\mathrm{p}<0.0001)$ and $0.133(\mathrm{p}<0.052)$. The syncope-only query saw no variation in HRI classified encounters throughout the heat season (mean: 328; min: 228; max: 404) or colder months (mean: 328; $\min : 261$; max: 404) with correlation coefficients of $0.238(\mathrm{p}=0.003)$ and $0.155(\mathrm{p}=0.024)$, respectively. Similarly, the dehydration-only query saw no variation in HRI classified encounters throughout the heat season (mean: 96; min: 58; max: 258) or colder months (mean: 94; min: 60; max: 160) with correlation coefficients of $0.596(\mathrm{p}<0.0001)$ and $-0.016(\mathrm{p}=0.822)$.

\section{Conclusions}

The CSTE HRI query proved to be a strong indicator for HRI, and the addition of terms associated with dehydration and syncope to the CSTE HRI query weakened the correlation with temperature. Compared to the original CSTE HRI query, the added terms yielded a $4934 \%$ increase in HRI classified encounters during the heat season; however, these were likely due to causes other than HRI -- adding the extra terms resulted in a weaker correlation with temperature. Additionally, the comparative analysis showed that, with the added terms, the volume of HRI encounters was larger during colder months than hotter months suggesting misclassification of non-HRI illnesses. Surveillance of HRI has proven to be difficult because many of the HRI symptoms are too commonly associated with non-HRI conditions which would explain the weaker correlations when adding additional chief 
complaints associated with HRI. In conclusion, the CSTE syndrome definition for HRI proved to be the most robust query for HRI during the heat season. Case counts of HRI are difficult due to symptom overlap with many other medical conditions. However, syndromic surveillance using the CSTE HRI query is useful for trend analysis in near real-time during heat events.

\section{References}

1. Council of State and Territorial Epidemiologists. Heat-Related Illness Syndrome Query: A Guidance Document for Implementing Heat-Related Illness Syndromic Surveillance in Public Health Practice. Version 1.0. 2016 Sep. 12 p.

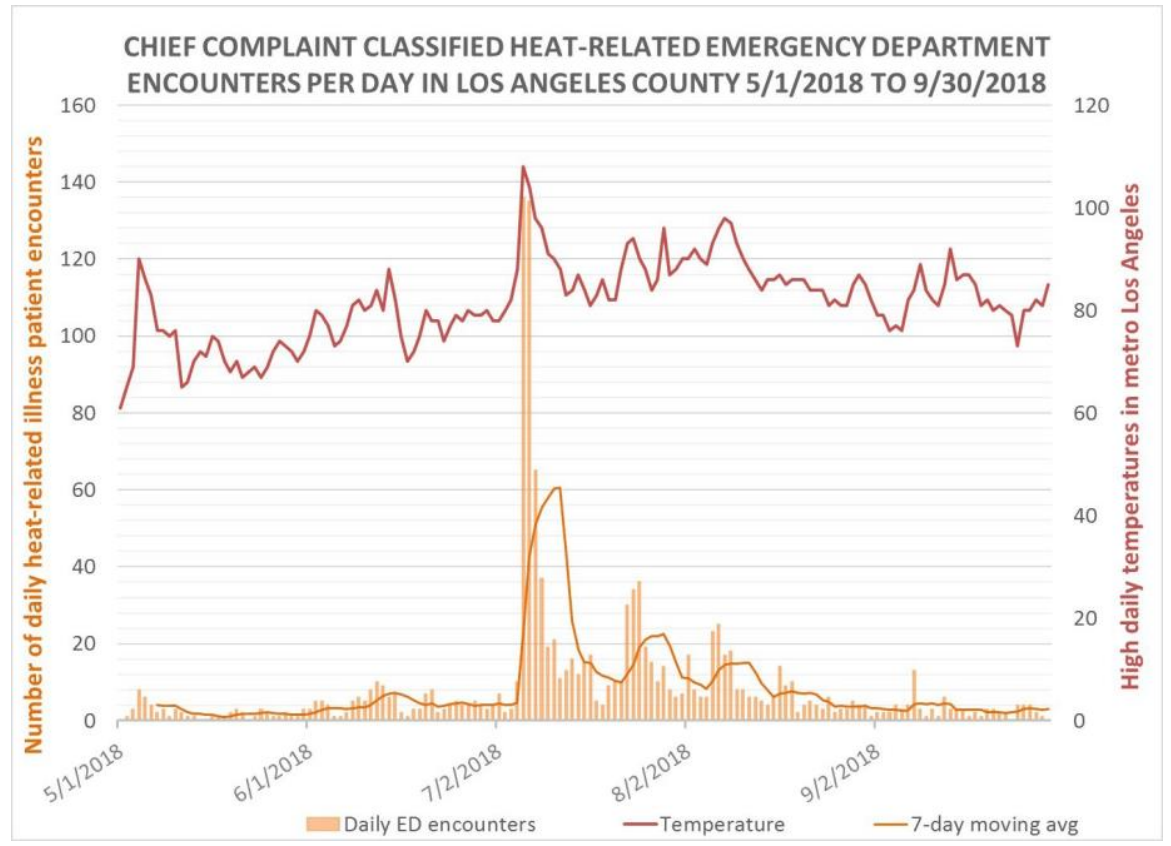

Figure 1. Heat-related ED visits, defined by CSTE's HRI syndrome definition, per day in Los Angeles County during the heat season from $5 / 1 / 2018$ to $9 / 30 / 2018$ 


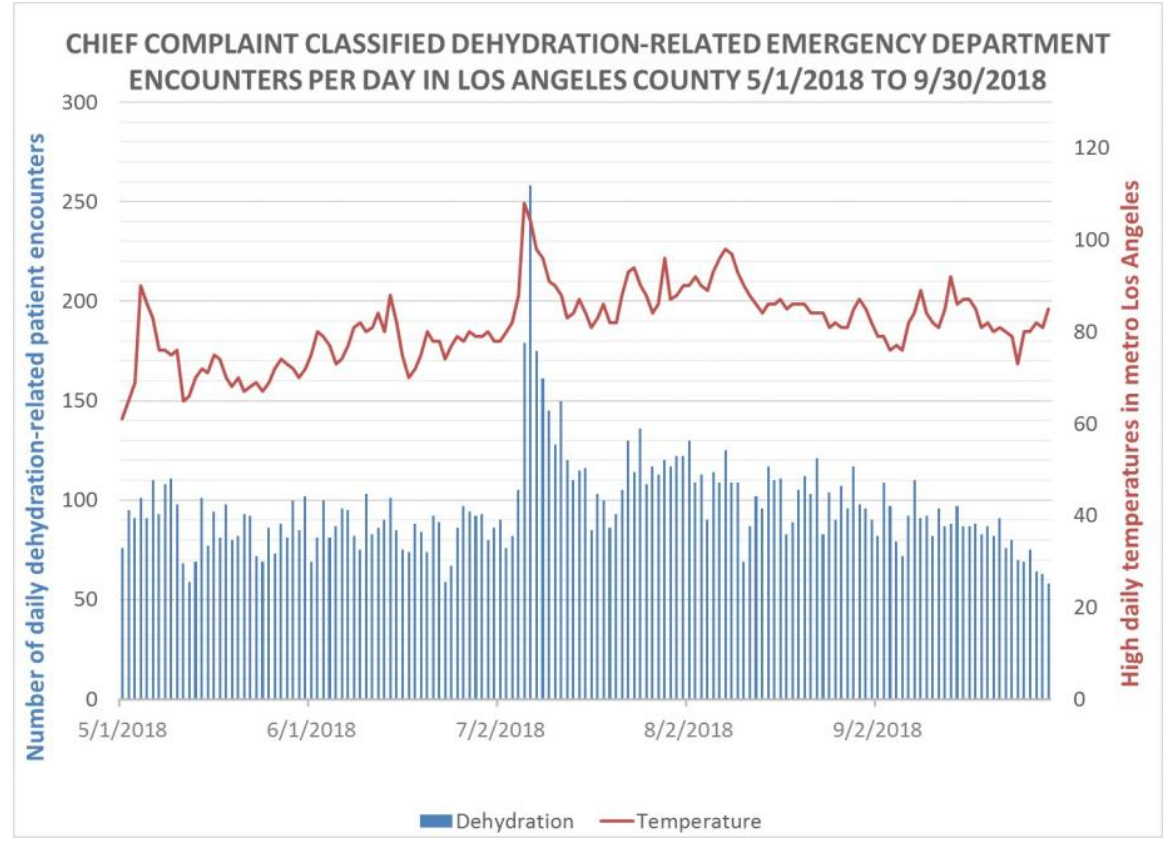

Figure 2. Dehydration-related ED visits per day in Los Angeles County during the heat season from 5/1/2018 to 9/30/2018

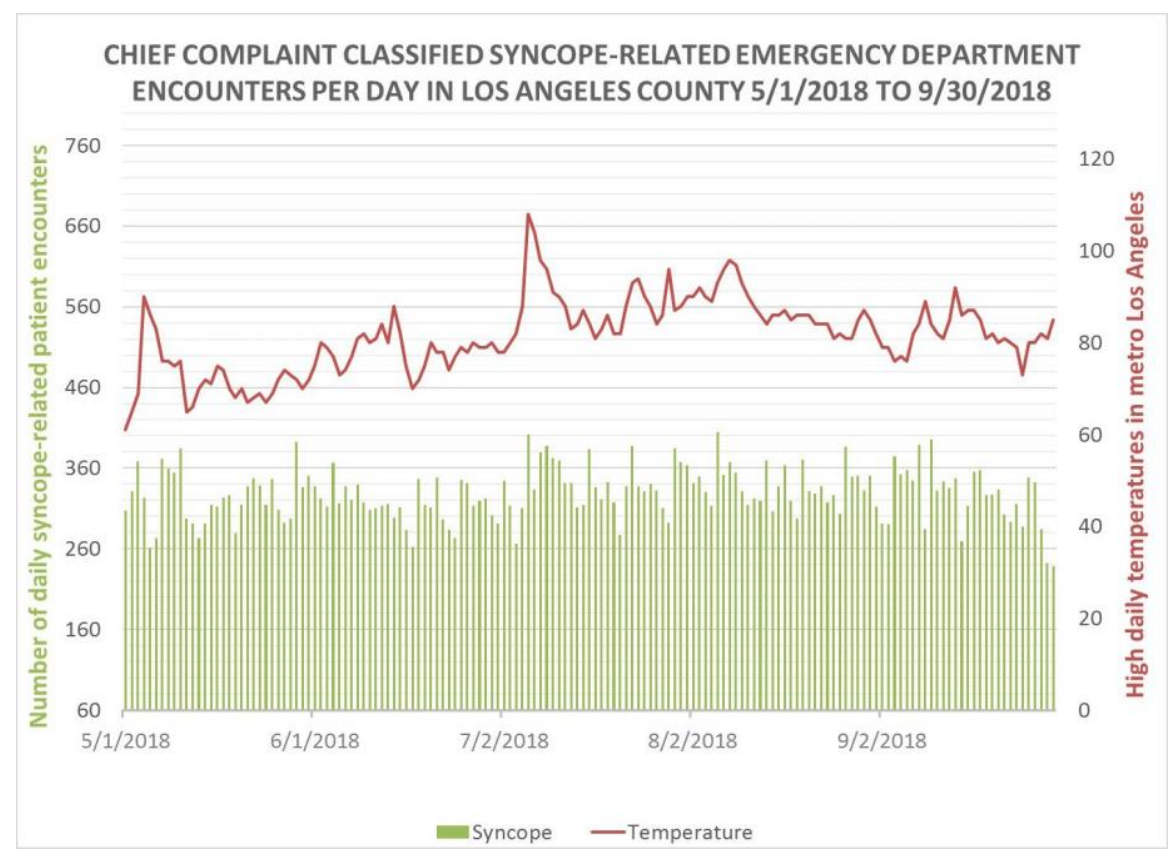

Figure 3. Syncope-related ED visits per day in Los Angeles county during the heat season from 5/1/2018 to 9/30/2018 